\title{
Thermal and field-induced transitions in ferroquadrupolar Kondo systems
}

\author{
Frederic Freyer $\odot,{ }^{1}$ SungBin Lee, ${ }^{2}$ Yong Baek Kim, ${ }^{3}$ Simon Trebst $\odot,{ }^{1}$ and Arun Paramekanti $\odot{ }^{3, *}$ \\ ${ }^{1}$ Institute for Theoretical Physics, University of Cologne, 50937 Cologne, Germany \\ ${ }^{2}$ Department of Physics, Korea Advanced Institute of Science and Technology, Daejeon 34141, Korea \\ ${ }^{3}$ Department of Physics, University of Toronto, Toronto, Ontario, Canada M5S 1A7
}

(Received 2 April 2020; revised 5 July 2020; accepted 7 July 2020; published 31 July 2020)

\begin{abstract}
Recent experiments have examined the impact of a magnetic field on ferroquadrupolar orders in the intermetallic Kondo material $\operatorname{PrTi}_{2} \mathrm{Al}_{20}$. Motivated by this, we use extensive Monte Carlo simulations to study a diamond lattice $X Y$ model of non-Kramers pseudospin-1/2 $\operatorname{Pr}^{3+}$ moments which crucially incorporates three-spin interactions. This model supports a thermal $Z_{3}$ Potts ordering transition upon cooling from the paramagnetic phase into the ferroquadrupolar phase. An applied magnetic field along the [110] direction leads to a thermal Ising transition out of the quadrupolar ordered phase. A magnetic field along the [001] direction leads to only thermal crossovers, but supports a spinodal transition out of metastable domains which could be strongly pinned by coupling to elastic lattice deformations. We propose noise measurements as a potential probe to "hear" the spinodal transition. Our work highlights the importance of multispin interactions in Kondo materials near the small-to-large Fermi surface transition.
\end{abstract}

DOI: 10.1103/PhysRevResearch.2.033176

\section{INTRODUCTION}

The famous Doniach picture of Kondo lattice compounds suggests a scenario for the small-to-large Fermi surface (FS) transition in Kondo lattice materials. In this framework, weak Kondo coupling leads to two-spin Ruderman-Kittel-KasuyaYosida (RKKY) interactions which drive rare-earth local moment ordering and a small FS, while strong Kondo coupling leads to the local moments hybridizing with the conduction electrons resulting in a heavy Fermi liquid with a large FS [1-6]. While there has been important work in understanding this physics for materials with local dipole moments [4,5,7-10], there is considerably less understanding of higher multipolar orders [11-18].

Recently, there has been significant experimental progress in unveiling the rich phase diagram of the cubic rare-earth intermetallics $\operatorname{Pr}(\mathcal{T})_{2} \mathrm{Al}_{20}(\mathcal{T}=\mathrm{Ti}, \mathrm{V})$ and $\operatorname{PrIr}_{2} \mathrm{Zn}_{20}$ [19-36] which feature $\operatorname{Pr}^{3+}$ local moments coupled to conduction electrons [2-6]. The complex multipolar orderings and superconductivity in these compounds may be tuned by the choice of transition metal ion or pressure. Understanding the broken-symmetry states and phase transitions in such multipolar Kondo materials remains a largely open issue.

One basic question which arises when one confronts the plethora of broken-symmetry states in Kondo materials is whether one needs to go beyond the simple two-spin RKKY model in modeling the effective interaction between local

\footnotetext{
*arunp@physics.utoronto.ca

Published by the American Physical Society under the terms of the Creative Commons Attribution 4.0 International license. Further distribution of this work must maintain attribution to the author(s) and the published article's title, journal citation, and DOI.
}

moments. Indeed, as the Kondo coupling in heavy fermion materials increases, we expect that multispin interactions can arise from higher-order perturbation theory beyond the simple RKKY limit. One setting in which such multispin interactions have been investigated extensively is in the vicinity of Mott transitions in quasi-two-dimensional organic materials [37-41], where it has been shown to potentially stabilize exotic quantum spin liquids. From this viewpoint, we expect multispin interactions to also emerge naturally in Kondo materials if we view the large-to-small FS transition as an orbital-selective Mott transition of the $f$ electrons [42,43]. The impact of such couplings has only recently been investigated in multipolar Kondo systems [44-47], although there has been some suggestive previous work in dipolar Kondo materials [48,49]. Given this, we ask the following questions. Are there any heavy fermion multipolar systems where multispin couplings play a role? Can such interactions lead to observable signatures?

We address these questions in the context of recent experiments on the $\operatorname{Pr}(\mathcal{T})_{2} X_{20}$ family of materials, where the $\mathrm{Pr}^{3+}$ ions feature a non-Kramers ground-state doublet, which acts as a pseudospin-1/2 degree of freedom on the diamond lattice [22,23]. As discussed in the literature, two components of this pseudospin carry a quadrupolar moment while the third component describes an octupolar moment $[23,50]$. In this paper we focus on $\operatorname{PrTi}_{2} \mathrm{Al}_{20}$, which has been proposed to host a ferroquadrupolar $\left(F_{\mathcal{Q}}\right)$ ordered ground state $[19,20,22,28,33]$ below $T_{Q} \approx 2 \mathrm{~K}$, well before the system enters a low-temperature superconducting state with transition temperature $T_{\mathrm{SC}} \approx 0.2 \mathrm{~K}$.

Recent experiments have studied the nontrivial impact of a magnetic field on this ferroquadrupolar ordered state, discovering a strong dependence of the response on the field direction [33,51,52]. For a magnetic field along the [111] direction, the quadrupolar transition appears nearly unaffected, 
while there appear to be distinct field-induced transitions for fields along the [001] and [110] directions. We argue here that an appropriate low-energy microscopic model for this material must necessarily include three-spin interactions and that it reveals itself via the impact of a magnetic field.

Our key results are the following. We show that the model pseudospin-1/2 Hamiltonian for local $\mathrm{Pr}^{3+}$ moments must include crucial symmetry-allowed three-spin couplings. We use classical Monte Carlo (MC) simulations to study the ordered states, thermal fluctuations, and the impact of a magnetic field in this model. We uncover thermal and field-induced phase transitions and crossovers which are qualitatively consistent with experimental observations. However, our model does not display a subset of field-induced transitions which have been inferred from certain experiments [51]. We argue here that such experiments could potentially probe spinodal transitions out of metastable ground states; such metastable states do exist in the model and may be rendered visible by the strong coupling between the quadrupolar order and elastic lattice deformations. We propose that noise measurements could be used to "hear" such spinodal transitions. While our work here focuses on $\operatorname{PrTi}_{2} \mathrm{Al}_{20}$, our main results are broadly applicable to ferroquadrupolar orders in diverse materials.

\section{MODEL}

We consider a simple low-energy diamond lattice model for the pseudospin-1/2 non-Kramers doublets, ignoring higher crystal field levels which are split off by a relatively big energy scale $\sim 50 \mathrm{~K}$, which is much larger than the observed ferroquadrupolar transition temperature. The pseudospin Hamiltonian we propose takes the form

$$
\begin{aligned}
H_{0}= & -J_{1} \sum_{\langle i j\rangle} \vec{\tau}_{i}^{\perp} \cdot \vec{\tau}_{j}^{\perp}-i \frac{\Gamma}{2} \sum_{\langle i j k\rangle}\left(\tau_{i}^{+} \tau_{j}^{+} \tau_{k}^{+}-\text {H.c. }\right) \\
& -\alpha \sum_{i}\left[\sqrt{3}\left(B_{x}^{2}-B_{y}^{2}\right) \tau_{i}^{x}+\left(3 B_{z}^{2}-B^{2}\right) \tau_{i}^{y}\right],
\end{aligned}
$$

where $\vec{\tau}^{\perp} \equiv\left(\tau_{x}, \tau_{y}\right)$ defines pseudospin-1/2 Pauli matrices and H.c. refers to the Hermitian conjugate. We denote nearestneighbor pairs by $\langle i j\rangle$, while the notation $\langle i j k\rangle$ refers to shortest site triplets on the diamond lattice as illustrated in Fig. 1. Our notation for the spin operators follows Refs. $[44,45]$ and differs from that used in some of the literature [51-53]. In our convention, $\left\langle\tau^{x}\right\rangle$ corresponds to $O_{22}$ order, while $\left\langle\tau^{y}\right\rangle$ refers to $O_{20}$ order, where $O_{22} \propto \sqrt{3}\left(\mathcal{J}_{x}^{2}-\mathcal{J}_{y}^{2}\right)$ and $O_{20} \propto$ $\left(3 \mathcal{J}_{z}^{2}-\mathcal{J}^{2}\right)$ are the standard Steven's operators written in terms of the total angular momentum $\overrightarrow{\mathcal{J}}$ of the $\operatorname{Pr}^{3+}$ ion.

An easy-plane interaction with $J>0$ is appropriate to describe ferroquadrupolar $X Y$ order in $\operatorname{PrTi}_{2} \mathrm{Al}_{20}$. The magnetic field $\boldsymbol{B}=\left(B_{x}, B_{y}, B_{z}\right)$ couples to the pseudospin at $O\left(B^{2}\right)$. This arises within second-order perturbation theory $[53,54]$ via intermediate states involving higher crystal field multiplets, with $\alpha>0$. Most importantly, the term $\Gamma>0$ is the simplest symmetry-allowed three-spin interaction which breaks the $X Y$ symmetry and leads to a $Z_{3}$ clock anisotropy. While such clock terms have been previously discussed within Landau theory $[45,53,54]$, there can be no such single-site clock anisotropy term for pseudospin-1/2 models. Hence, the

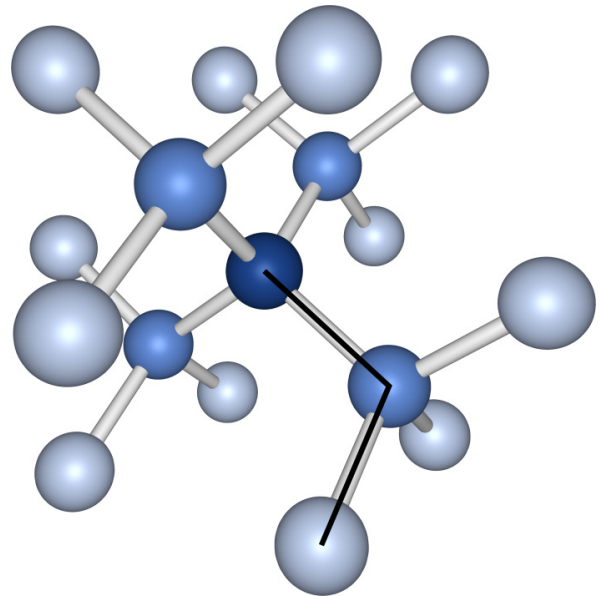

FIG. 1. Cutout of a diamond lattice with nearest-neighbor bonds $\left(J_{1}\right)$ drawn in white. The three-site triplets of the $\Gamma$ term are constructed by two adjacent nearest-neighbor bonds as exemplified by the black line.

clocklike anisotropy for pseudospin- $1 / 2$ cases must necessarily arise from multisite couplings at the lattice scale. We note that this multispin interaction allows for the $\tau_{z}$ eigenvalue to change in steps of \pm 3 , which cannot arise in any RKKY-type two-spin exchange model.

Our motivation here is to understand the ordered phases and thermal transitions of such quadrupolar spin models. We will thus focus on a mean-field theory and large-scale classical MC simulations of this model replacing $\vec{\tau}^{\perp}$ by a classical $X Y$ vector spin. It would be interesting in the future to examine the impact of quantum spin fluctuations in this model.

\section{MEAN-FIELD THEORY}

At zero temperature and at the mean-field level, we replace uniformly $\tau_{i}^{+}=e^{i \theta}$, which leads to an energy per spin

$$
\begin{aligned}
e_{\mathrm{mf}}= & -2 J_{1}+6 \Gamma \sin 3 \theta \\
& -\alpha\left[\sqrt{3}\left(B_{x}^{2}-B_{y}^{2}\right) \cos \theta+\left(3 B_{z}^{2}-B^{2}\right) \sin \theta\right] .
\end{aligned}
$$

The magnetic field thus competes with the $\Gamma$ term, allowing us to probe the impact of the reduction of symmetry from $\mathrm{U}(1)$ to $Z_{3}$. We see that applying a field in the [111] direction will not couple at all to the quadrupolar field. A magnetic field along the [110] direction gives $\alpha B^{2} \sin \theta$, while a field along the [001] direction gives $-2 \alpha B^{2} \sin \theta$. Figure 2 plots the energy landscape as a function of $\theta$ and $B$ for the latter two field directions.

As can be seen from Fig. 2(a), a magnetic field along [001] favors $\theta=\pi / 2$, while the two other zero-field minima become metastable minima at $\theta=(7 \pi / 6-\delta, 11 \pi / 6+$ $\delta)$, where $\delta \approx \sqrt{3} \alpha B^{2} /\left(54 \Gamma-\alpha B^{2}\right)$ for weak fields. These metastable minima lie at an energy approximately $3 \alpha B^{2}$ above the ground state, vanishing at a field $B_{\mathrm{sp}}=\sqrt{9 \Gamma / \alpha}$ which marks a field-induced spinodal transition.

For a [110] field, as can be seen from Fig. 2(b), there are two energy minima which lie at $\theta=\left(7 \pi / 6+\delta^{\prime}, 11 \pi / 6-\right.$ $\left.\delta^{\prime}\right)$, where we find that $\delta^{\prime} \approx \sqrt{3} \alpha B^{2} /\left(108 \Gamma+\alpha B^{2}\right)$ for small $B$. The third zero-field minimum becomes a metastable 

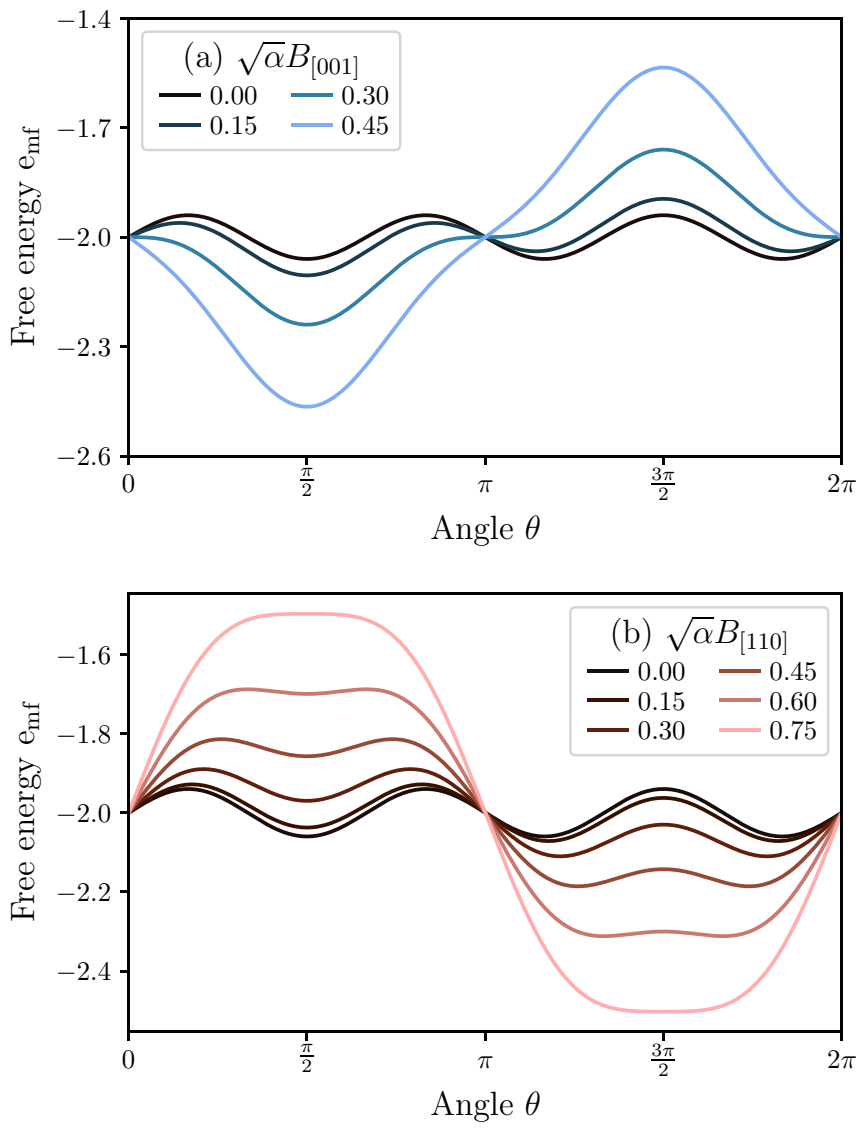

FIG. 2. Sketch of the mean-field free energy per spin (2) for $J_{1}=1$ and $\Gamma=0.01 J_{1}$, plotted versus $\theta$ for increasing magnetic field along the (a) [001] and (b) [110] directions.

minimum at $\theta=\pi / 2$, which lies at an energy approximately $3 \alpha B^{2} / 2$ above the global minima. This will convert the thermal $Z_{3}$ clock transition into an Ising transition since the three ground states of the $Z_{3}$ clock model have been reduced to just two degenerate ground states. Eventually, the two minima merge at $B^{\star}$, which marks the end point of the Ising transition, where $B^{\star}=\sqrt{54 \Gamma / \alpha}$. The metastable minimum at $\theta=\pi / 2$ persists until $B^{\star}$.

We thus expect that for the [001] field direction, the field should immediately round off the $Z_{3}$ thermal transition into a crossover by selecting one of the three ground states. For the [110] direction, on the other hand, we expect the $Z_{3}$ thermal transition to convert into an Ising transition for arbitrarily weak fields, with the Ising transition vanishing above a critical field $B^{\star}$.

\section{METASTABLE MINIMA}

The reason why the metastable minima in Fig. 2 may be important to explore in this system is the following. Imagine we consider starting from a paramagnetic state at high temperature. When we cool below the ferroquadrupolar transition $T_{Q}$ at zero field, we would end up having different $Z_{3}$ domains of a typical size $L(T)$ at some temperature $T<$ $T_{c}$. Ferroquadrupolar order in this system induces a lattice distortion, which arises from coupling an elastic distortion field $\vec{\varphi}$ to the quadrupolar degree of freedom, which can be understood via a coarse-grained Hamiltonian

$$
H=H_{0}+\frac{1}{2} \kappa \int d^{3} \mathbf{r} \vec{\varphi}(\mathbf{r}) \cdot \vec{\varphi}(\mathbf{r})-\lambda \int d^{3} \mathbf{r} \vec{\varphi}(\mathbf{r}) \cdot \vec{\tau}^{\perp}(\mathbf{r}),
$$

where $\vec{\tau}^{\perp}(\mathbf{r})$ is the coarse-grained quadrupolar order parameter and $\lambda$ denotes the magnetoelastic coupling. The twocomponent elastic distortion field $\vec{\varphi}$ may be written in terms of the elastic strain tensor $\varepsilon$ as $\varphi_{x}=\varepsilon_{x x}-\varepsilon_{y y}$ and $\varphi_{y}=\left(2 \varepsilon_{z z}-\right.$ $\left.\varepsilon_{x x}-\varepsilon_{y y}\right) / \sqrt{3}$. The impact of quadrupolar order will thus be to produce a small nonzero lattice distortion $\vec{\varphi}$. This elastic deformation along different directions in the different domains will tend to collectively pin the local order. Thus, we see that while an applied [001] field will favor a single domain, we have to thermally excite the system out of the metastable domains in order to get to the true equilibrium state. If thermal fluctuations are not significant at low temperature, then such domains might get stuck until we reach a threshold field corresponding to a mean-field spinodal transition; this effect may reveal itself in certain experiments.

\section{MONTE CARLO SIMULATIONS}

We have carried out extensive classical MC simulations of the Hamiltonian $H_{0}$ from Eq. (1). While standard MC updates sufficed to explore the equilibrium phase diagram via measurements of the specific heat and ferroquadrupolar $X Y$ order parameter $\vec{M}_{\mathrm{FQ}}=\sum_{i} \vec{\tau}_{i}^{\perp}$, exploring the metastable transitions required us to choose a special update engineered to probe the free energy as a function of the angle $\theta$ of $\vec{M}_{\mathrm{FQ}}$. The update involves a local update conserving the direction of $\vec{M}_{\mathrm{FQ}}$ and a global update jumping between two angles. Combining multiple such simulations at slowly varying angles (typically $\Delta \theta=2 \pi / 1080)$, we recover the relative weights between them and ultimately estimate the free-energy landscape. Further details on this procedure are provided in the Appendix. Simulations were typically done with $2 \times 10^{6}$ thermalization and $8 \times 10^{6}$ measurement sweeps for a linear system size of $L=9$ (corresponding to $2 L^{3}=1458$ spins) when mapping out the phase diagram, and for a linear system size $L=6$ when studying the metastable regions. The numerical simulations were performed on the CHEOPS cluster at RRZK Cologne.

\section{A. Zero-field phase diagram}

The phase diagram of the model with $J_{1}=1$ in the absence of any magnetic field is shown in Fig. 3(a). Based on a finitesize scaling analysis of specific heat data (for $L=6, \ldots, 24$ ), we find a sharp thermodynamic phase transition at $T=T_{Q}$ as illustrated in Fig. 4 and indicated by the solid transition line in Fig. 3.

For $\Gamma=0$ we expect this transition to be in the universality class of the three-dimensional $X Y$ model, and the corresponding ferroquadrupolar order parameter $m_{\mathrm{FQ}}=\left\langle\left|\vec{M}_{\mathrm{FQ}}\right|\right\rangle / N$ indeed continuously vanishes when we heat above the transition temperature $T_{Q} \approx 1.3 J_{1}$ as seen from Fig. 3(b). When we turn on $\Gamma \neq 0$, the clock anisotropy suppresses fluctuations and enhances $T_{Q}$; furthermore, the transition becomes first order, 

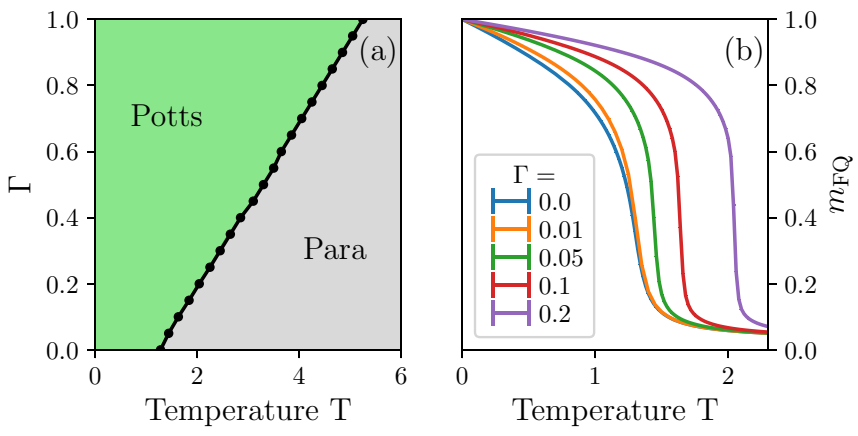

FIG. 3. (a) Zero-field phase diagram of the Hamiltonian $H_{0}$, with $J_{1}=1$, as a function of temperature $T$ and varying clock anisotropy $\Gamma$. (b) Ferroquadrupolar order parameter $m_{\mathrm{FQ}}=\left\langle\left|\vec{M}_{\mathrm{FQ}}\right|\right\rangle / N$ as a function of $T$ for horizontal cuts through the phase diagram in (a). Here $m_{\mathrm{FQ}}$ vanishes continuously for $\Gamma=0$, but becomes discontinuous for $\Gamma \neq 0$; this discontinuity becomes more pronounced for large $\Gamma$.

as is confirmed by the increasingly sharp and discontinuous drop of $m_{\mathrm{FQ}}$ across $T_{Q}$. The formation of the three clock states below this transition is nicely visible in our Monte Carlo simulations, as illustrated in Fig. 5.

\section{B. Impact of a nonzero magnetic field}

Figure 6 shows the impact of a magnetic field on the phase diagram for fixed $J_{1}=1$ and $\Gamma=0.01 J_{1}$ (see also Fig. 7). The top and bottom halves in this diagram correspond to fields along the [001] and [110] directions, respectively. In the following section, we argue that $\Gamma / J_{1} \sim 10^{-3}$ for experiments on $\operatorname{PrTi}_{2} \mathrm{Al}_{20}$; however, the numerical simulations are more challenging for such small $\Gamma$. We thus choose to work with a larger $\Gamma$ in the MC simulations. The magnetic field required to induce the relevant transitions or crossovers scales as proportional to $\sqrt{\Gamma}$ as indicated by mean-field theory. We can thus use our MC results, with suitable scaling, to make useful comparisons with experiment.

As expected, a sufficiently large magnetic field leads to a crossover temperature scale since it favors a single free-energy minimum as seen from the free-energy plots for I and IV in the left panel, where the color at the bottom depicts the favored angle $\theta$. This crossover temperature $T^{*}$, indicated by the dotted line, is detected in our MC simulations as a broad hump in the specific heat which does not scale with system size (based on simulations done for linear system sizes $L=6,9,12,18,24)$.

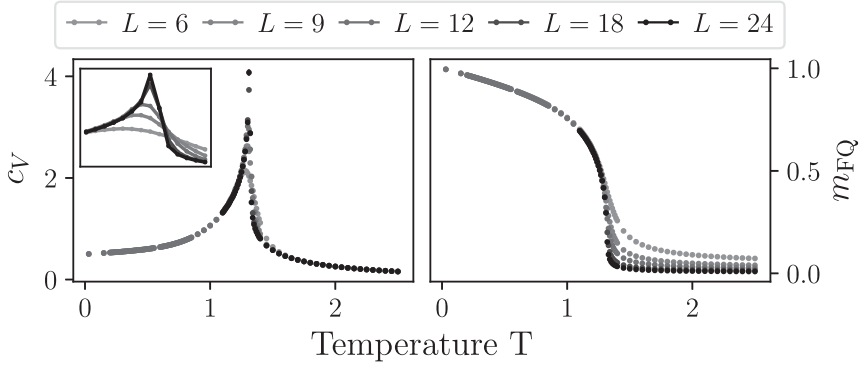

FIG. 4. Finite-size scaling of the zero-field specific heat $c_{V}$ and ferroquadrupolar order parameter $m_{\mathrm{FQ}}$ at $\Gamma=0.01$.

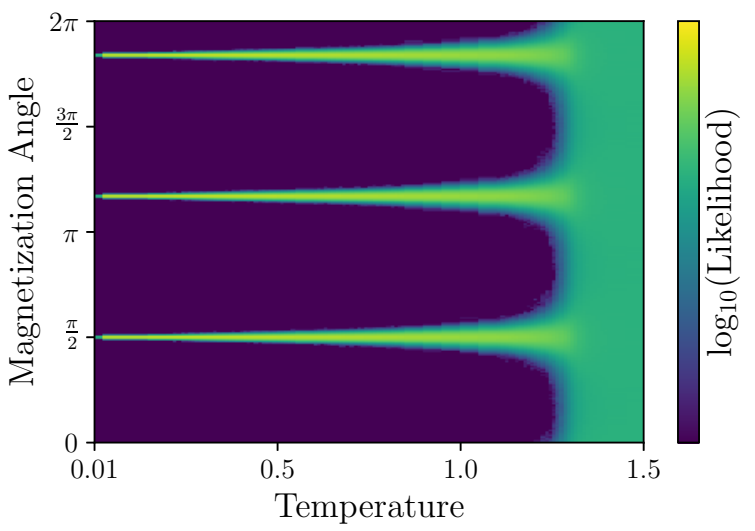

FIG. 5. Probability of different magnetization angles for $\Gamma=$ 0.01 and $L=12$ in the zero-field system as a function of temperature (in units of $J_{1}$ ). At high temperature $T>T_{Q}$, the distribution is broad, while the locking to the $Z_{3}$ clock order becomes increasingly visible as we go below $T_{Q} \sim 1.3 J_{1}$.

At low field, the [110] direction leads to an Ising transition, denoted by the solid black line, into a phase where there are two degenerate minima as seen from the free-energy plot III in the left panel. Different MC runs (initialized with a random state) in this regime lead to the system ending up in one or the other minimum, which is depicted by the colors in phase III with corresponding $\theta$ values shown in the left panel.

For the [001] field direction, even at low field, a single free-energy minimum is selected as seen from the left panel II. The dashed line indicates the crossover field beyond which the metastable free-energy minima in the left panel II disappear;
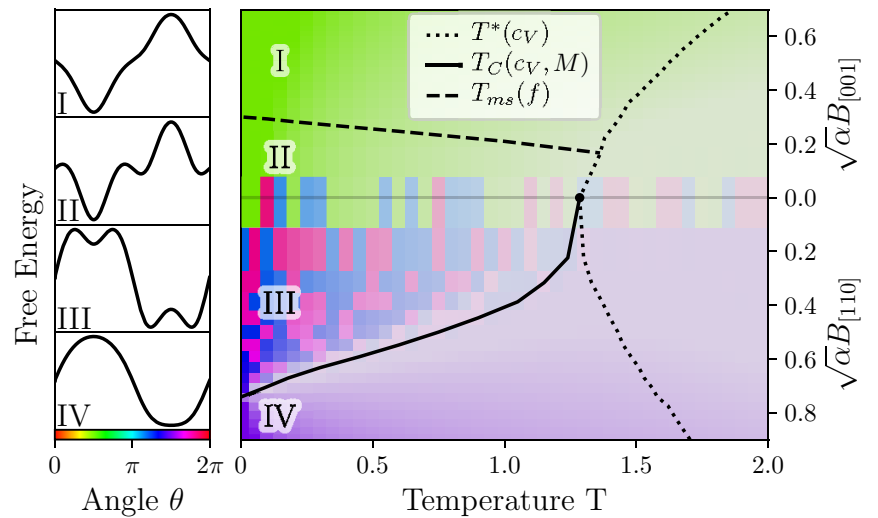

FIG. 6. Phase diagram for $B \neq 0$ along the [001] direction (upper half) and [110] direction (lower half) for fixed $J_{1}=1$ and $\Gamma=$ $0.01 J_{1}$. The left panel indicates the free energy in the different low-temperature regimes shown in the phase diagram. The colors in the phase diagram indicate the (dominant) angle $\theta$ as depicted at the bottom of the left panel. The solid line shows the Ising phase transition $T_{C}$ for the [110] field direction which is extracted from specific heat $c_{V}$ and order parameter $M$, dotted lines depict thermal crossovers $T^{*}$ obtained from the specific heat $c_{V}$, and the dashed line shows the field where the metastable $(\mathrm{ms})$ minima in regime II vanish (see left panel), which we extract from free-energy $(f)$ calculations as explained in the text and Appendix. 


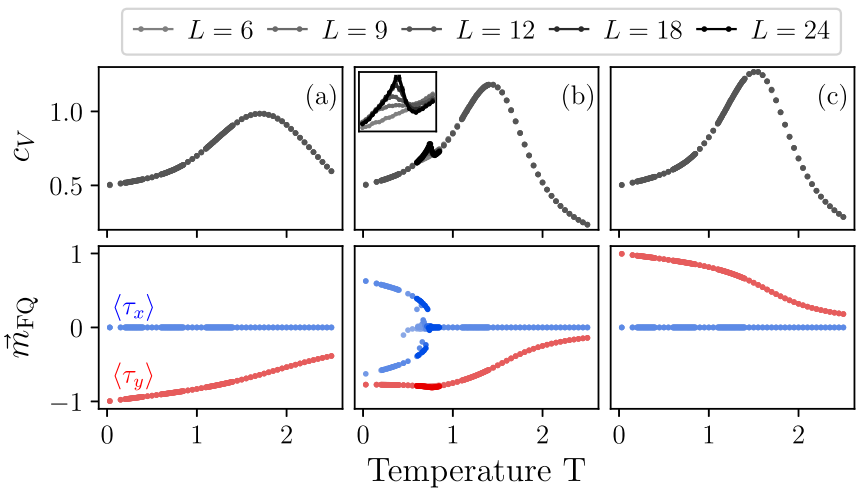

FIG. 7. Finite-size scaling of specific heat $c_{V}$ and site-averaged magnetization $\vec{m}_{\mathrm{FQ}}=\left(\left\langle\tau_{x}\right\rangle,\left\langle\tau_{y}\right\rangle\right)$ versus temperature for fixed magnetic fields, corresponding to horizontal cuts through the phase diagram in Fig. 6: (a) $\sqrt{\alpha} B_{[110]}=0.9$, which accesses regime IV going through a thermal crossover; (b) $\sqrt{\alpha} B_{[110]}=0.5$, which enters the broken-symmetry phase in regime III via a highertemperature crossover and a lower-temperature true phase transition; and (c) $\sqrt{\alpha} B_{[001]}=0.4$, which passes through regime I via a thermal crossover.

this corresponds to the spinodal transition discussed above from the perspective of mean-field theory.

The results from our extensive MC simulations are thus broadly consistent with expectations based on mean-field theory, but with thermal fluctuations suppressing the magnetic field scale required to induce the observed phase transitions and crossovers. We next turn to the experimental implications of this phase diagram.

\section{EXPERIMENTAL IMPLICATIONS}

Our classical MC simulations show that the zero-field ferroquadrupolar transition for $\Gamma=0$ occurs at $T_{Q} \approx 1.3 J_{1}$. As $\Gamma$ increases, $T_{Q}$ increases and the transition becomes more visibly first order, consistent with the behavior of the three-dimensional $Z_{3}$ clock (or equivalently three-state Potts) model. Since the experiments $[19,22]$ detect what appears to be a nearly continuous thermal transition at $T_{Q} \approx 2.2 \mathrm{~K}$, we assume $\Gamma \ll J_{1}$. We thus use the value of $T_{Q}$ at $\Gamma=$ 0 to roughly estimate $J_{1} \sim 1.7 \mathrm{~K}$. Microscopic calculations [53] using the measured crystal field levels [19,22] yield $\alpha=\left(g \mu_{B}\right)^{2}\left(7 / 3 E_{4}-1 / E_{5}\right)$, where $g=4 / 5$. For $\operatorname{PrTi}_{2} \mathrm{Al}_{20}$, the relevant excited crystal field levels [22] lie at $E_{4} \approx 65 \mathrm{~K}$ and $E_{5} \approx 107 \mathrm{~K}$. This yields $\alpha \approx 0.008 \mathrm{~K} / \mathrm{T}^{2}$. Assuming the Ising transition for the [110] field direction [51,52] disappears at $B^{\star} \sim 3 \mathrm{~T}$, we are led to estimate $\Gamma=\alpha B^{\star 2} / 54 \approx 10^{-3} \mathrm{~K}$ so that indeed $\Gamma \ll J_{1}$. The spinodal transition for the [001] field direction is then expected to occur around $B_{\mathrm{sp}} \sim 1.2 \mathrm{~T}$.

Recently, transport, magnetization, and ${ }^{27} \mathrm{Al}$ nuclear magnetic resonance (NMR) experiments [51,52] have been used to further explore the phase diagram of $\operatorname{PrTi}_{2} \mathrm{Al}_{20}$. At high fields $B \gtrsim 4 \mathrm{~T}$ for both the [001] and [110] directions, there is a significant enhancement of the magnetization [52] upon cooling below $T \lesssim 3 \mathrm{~K}$. However, for low fields $B \lesssim 2 \mathrm{~T}$, this strong enhancement is absent. The bulk magnetization is given by the field derivative of the free energy, $\vec{m}=-\partial F / \partial \vec{B}$ (note that this is not $m_{\mathrm{FQ}}$ ). For both field directions, this is given (up to a sign) by $|\vec{m}| \propto B\left\langle\tau_{y}\right\rangle$. For sufficiently high fields, we expect the system to evolve from $\left\langle\tau_{y}\right\rangle \sim 0$ for high temperature to a nearly polarized value $\left|\left\langle\tau_{y}\right\rangle\right| \sim 1$ at low temperature so that there would be a significant increase in $|\vec{m}|$ below a crossover temperature. By contrast, in the presence of metastable domains which we expect at low fields, $\left\langle\tau_{y}\right\rangle$ would be greatly reduced via averaging over the domains, since $\left\langle\tau_{x}\right\rangle$ will also be nonzero in some domains. This leads to the suppression of the bulk magnetization in low fields so that the sharp increase upon cooling seen at higher fields will now be absent, in qualitative agreement with the data. Furthermore, NMR measurements of the Knight shift [52] are consistent with the bulk susceptibility from the magnetization measurements at high field, but in disagreement at low fields; this disagreement might also indirectly signal the presence of an inhomogeneous domain structure at low fields. The presence of domains is predicted to lead to NMR line splittings, or to inhomogeneous line broadening if the splitting is weak. This expectation is qualitatively borne out from the experimental data [52], but a detailed theoretical understanding needs further analysis using the microscopic hyperfine couplings. Scattering from such an inhomogeneous domain structure could partially contribute to the experimentally observed resistivity anomalies [51].

In order to estimate the typical linear dimension $L_{D}$ of $Z_{3}$ domains, we ask when the system with an average order parameter pointing along an $X Y$ angle $\theta$ would rather break up into domains of the discrete $Z_{3}$ order to save bulk anisotropy energy, governed by $\Gamma$, at the expense of a domain wall cost arising from $J_{1}$. Assuming a lattice constant $a$, we thus equate $6 \Gamma\left(L_{D} / a\right)^{3} \sim J_{1}\left(L_{D} / a\right)^{2}$ which, for $\Gamma / J_{1} \sim 10^{-3}$, leads to $L_{D} \sim 160 a$. This might be the size of typical domains we expect to get pinned by elastic lattice deformations.

One possible experimental route to further explore such a spinodal origin of the magnetization and transport anomalies could be noise spectroscopy. For instance, resistivity measurements in nanowires of high-temperature cuprate superconductors exhibit a telegraph noise, which has been attributed to fluctuating Ising nematic domains or charge stripe domains $[55,56]$. Similar field- and temperature-dependent resistivity noise measurements might be valuable to probe $Z_{3}$ domains in $\operatorname{PrTi}_{2} \mathrm{Al}_{20}$. Another possible experiment might be to detect the actual sound associated with the avalanche of domain rotations one expects near these metamagnetic transitions. Such experiments would be particularly useful in the low-field regime which is inaccessible for NMR.

Our proposal of strong spin-lattice coupling leading to field-induced anomalies is distinct from, but not entirely at odds with, a previously proposed explanation [52], which has considered the impact of additional field-dependent quadrupolar exchange couplings within an effective Landau theory. While the microscopic origin of this effect has been attributed to field-induced changes in the Fermi surface [52], and thereby the RKKY Kondo couplings, such terms may also occur if we incorporate field-dependent spin-phonon coupling and integrate out the phonons. The microscopic details of such a mechanism, as well as its connection with the metastable domain picture discussed here, remains a topic for future study.

In summary, understanding the nature of the fielddependent phase transitions and anomalies in $\operatorname{PrTi}_{2} \mathrm{Al}_{20}$ may 
help deepen our understanding of multipolar orders in heavy fermion materials. Finally, our work suggests that multispin interactions must play a broadly important role in Kondo materials.

\section{ACKNOWLEDGMENTS}

A.P. acknowledges funding from NSERC of Canada. S.T. and A.P. acknowledge partial funding from the DFG within CRC 1238 (project C02), Project No. 277146847. Y.B.K. was supported by the Killam Research Fellowship from the Canada Council for the Arts and NSERC of Canada. S.L. was supported by the KAIST startup and National Research Foundation Grant No. NRF-2017R1A2B4008097. F.F. is grateful to the Bonn-Cologne Graduate School of Physics and Astronomy for support. We acknowledge the computational resources provided by RRZK Cologne.

\section{APPENDIX: ALGORITHMS}

Equilibrium states of classical many-body systems can be probed by standard Monte Carlo simulations. For system which do not allow for efficient nonlocal (cluster) update, extended ensemble approaches such as simulated annealing and parallel tempering are often used to find the equilibrium state more efficiently and accurately. If, however, one is primarily interested in metastable states, then nonstandard procedures are often called for. In the following sections, we describe our problem-specific approach of resolving metastable states for model (1). Notably, our approach also allows us to probe the free energy as a function of angle $\theta$.

\section{Pair sampling}

We start by discussing a tailor-made update procedure for our model, which allows us to simultaneously sample two dominant angles of the $X Y$ order parameter and, by recording the number of sweeps spent on each, the relative probability between them. The procedure is split into two parts, a local update which preserves the $X Y$ order parameter angle $\theta$ and a global update which perform jumps between the two angles of interest. The latter is simply a global update rotating the whole spin configuration back and forth. The former is more complex and requires a more thorough discussion. As the local update is explicitly biased (by forcing $\theta$ to be constant), we present two algorithms and briefly discuss the effect of different biases on the derived free energy.

Just for the purpose of this Appendix, we introduce slightly convenient notation, defining the unit vector $\vec{S}_{i} \equiv \vec{\tau}_{i}^{\perp}$. The $X Y$ order parameter in a given configuration is then $\vec{M}_{\mathrm{FQ}}=$ $\sum_{i} \vec{S}_{i}, \hat{e}_{M}=\vec{M}_{\mathrm{FQ}} /\left|\vec{M}_{\mathrm{FQ}}\right|$ will be the direction of the $X Y$ order parameter, and $\hat{e}_{\perp}=\hat{e}_{z} \times \hat{e}_{M}$ is the direction perpendicular to it. If we want to sample configurations with fixed $\hat{e}_{M}$, i.e., a fixed angle in which the global $X Y$ order parameter points, we must use configuration updates $\left\{\vec{S}_{i}\right\} \rightarrow\left\{S_{i}^{\prime}\right\}$ such that $\sum_{i}\left(\vec{S}_{i}^{\prime}-\vec{S}_{i}\right) \cdot \hat{e}_{\perp}=0$. The first algorithm proceeds to do this in following steps.

1. Pick a random site $i$ and a new random spin $\vec{S}_{i}^{\prime}$. Compute $x=\left(\vec{S}_{i}^{\prime}-\vec{S}_{i}\right) \cdot \hat{e}_{\perp}$, which is the component in the $\hat{e}_{\perp}$ direction that must be compensated.

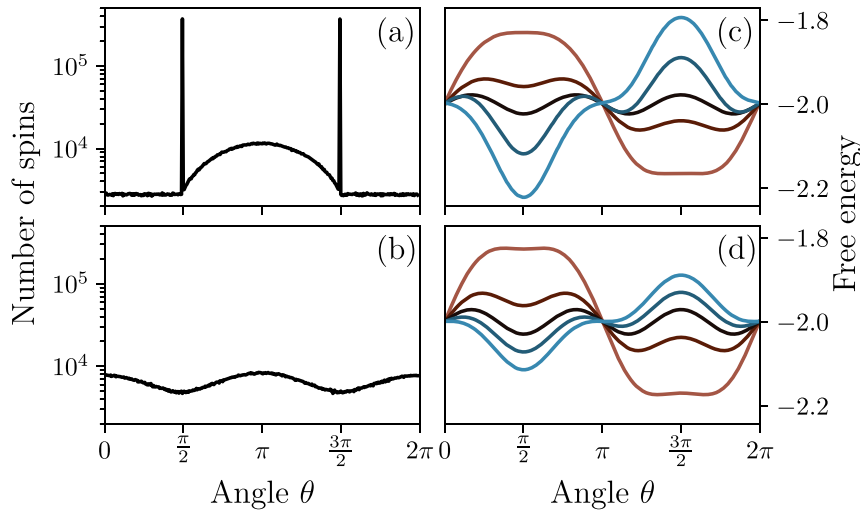

FIG. 8. Comparison between pair-sampling algorithms. The different algorithms are shown in rows, with the top row being the first algorithm and the bottom row being the second; in this plot, the temperature $T=1$ (in units of $J_{1}$ ). The left column shows a histogram of the proposed spins, where $\hat{e}_{M}=(\cos (\pi), \sin (\pi))$ is set for both. The right column shows the free-energy curves resulting from the respective updates for magnetic fields $\sqrt{\alpha} B \approx 0,0.28,0.49$ in the [110] direction and $\sqrt{\alpha} B \approx 0.2,0.32$ in the [001] direction.

2. Pick a random site $j$ which has not yet been chosen. Compute $\Delta x=\left(\vec{S}_{j}^{\prime}-\vec{S}_{j}\right) \cdot \hat{e}_{\perp}=\mp 1-\vec{S}_{j} \cdot \hat{e}_{\perp}$, which is the maximum compensation that can be achieved by setting $\vec{S}_{j} \rightarrow$ $\mp \hat{e}_{\perp}$ (for $x \gtrless 0$, respectively).

3. If $x^{\prime}=x-\Delta x=0$ or changes sign, the random spin flip can be fully compensated. Compute the necessary $\vec{S}_{j}^{\prime}$ and return every changed spin as a proposed update.

4. If $x^{\prime}$ has the same sign as $x$ the random spin flip cannot be compensated. Set $\vec{S}_{j} \rightarrow \mp \hat{e}_{\perp}, x \rightarrow x^{\prime}$, and go to step 2 , picking an additional (unique) site for the update.

We note that this algorithm comes with a strong localized bias because updates frequently include setting one or more spins $\vec{S} \rightarrow \pm \hat{e}_{\perp}$. Figure 8(a) shows a histogram of the proposed spins, making the bias obvious as two sharp peaks. The second algorithm is designed to avoid this bias. It includes the following steps.

1. Pick spins at two distinct random sites $\left\{i_{1}, i_{2}\right\}$ and two new random spin vectors $\left\{\vec{S}_{1}^{\prime}, \vec{S}_{2}^{\prime}\right\}$.

2. Compute combined vector length $a=\left|\vec{S}_{1}^{\prime}+\vec{S}_{2}^{\prime}\right|$ and combined component $b=\left(\vec{S}_{i_{1}}+\vec{S}_{i_{2}}\right) \cdot \hat{e}_{\perp}$ that must be compensated.

3. If $a>b$ a rotation $R$ can be found such that $\left\{R \vec{S}_{1}^{\prime}, R \vec{S}_{2}^{\prime}, \ldots\right\}$ keeps the $X Y$ order parameter direction $\hat{e}_{M}$ unchanged. Compute this rotation and return the rotated spins as a proposed update.

4. If $a<b$ we cannot find such a rotation. Add a new random spin $\vec{S}_{3}^{\prime}$ and a new (unique) random site $i_{3}$ to the collection of updated spins and sites and repeat from step 2 .

This update is weakly biased in the $\pm \hat{e}_{M}$ direction. As evident in Fig. 8(b), the distribution of proposed spins is much smoother.

\section{Computation of free energy}

The pair-sampling method allows us to fix two angles of the $X Y$ order parameter $\{\theta, \theta+\Delta \theta\}$. By counting the number of sweeps spent at each angle we can determine the relative 
weight between them

$$
\frac{Z(\theta+\Delta \theta)}{Z(\theta)}=\frac{N(\theta+\Delta \theta)}{N(\theta)} .
$$

By setting an initial value for $Z(0)$ we can compute successive $Z(\theta>0)$. From this we can derive the angle-resolved free energy $F(\theta)=-\ln [Z(\theta)] / \beta$. Note that this process becomes increasing expensive at low temperatures, requiring small $\Delta \theta$ and a large number of sweeps to get finite counts $N(\theta)>0$. The free energy per site $F(\theta) / N_{\text {site }}$ from the two algorithms is compared in Figs. 8(c) and 8(d); they show a very similar angle dependence, although there is some difference in the amplitude of the free-energy variation for a field applied along the [001] direction. The second algorithm with a smoother distribution of proposed updates is likely to be a better representation of the true free-energy curve. Comparing the computed result to the mean-field free energy, we find that the angle dependence is nearly identical; the Monte Carlo and mean-field curves match closely up to an overall $\sim 2.4$ scale factor, which reflects a renormalization of $\Gamma$ due to thermal fluctuation effects beyond mean-field theory.
[1] S. Doniach, Physica B+C 91, 231 (1977).

[2] M. A. Ruderman and C. Kittel, Phys. Rev. 96, 99 (1954).

[3] T. Kasuya, Prog. Theor. Phys. 16, 45 (1956).

[4] K. Yosida, Phys. Rev. 106, 893 (1957).

[5] S. G. Stewart, Rev. Mod. Phys. 56, 755 (1984).

[6] H. v. Löhneysen, A. Rosch, M. Vojta, and P. Wölfle, Rev. Mod. Phys. 79, 1015 (2007).

[7] Z. Fisk, J. Sarrao, J. Smith, and J. Thompson, Proc. Natl. Acad. Sci. USA 92, 6663 (1995).

[8] P. Coleman, C. Pépin, Q. Si, and R. Ramazashvili, J. Phys.: Condens. Matter 13, R723 (2001).

[9] P. Gegenwart, Q. Si, and F. Steglich, Nat. Phys. 4, 186 (2008).

[10] Q. Si and F. Steglich, Science 329, 1161 (2010).

[11] P. Morin, D. Schmitt, and E. D. T. De Lacheisserie, J. Magn. Magn. Mater. 30, 257 (1982).

[12] D. Cox, Phys. Rev. Lett. 59, 1240 (1987).

[13] D. Cox and A. Zawadowski, Exotic Kondo Effects in Metals: Magnetic Ions in a Crystalline Electric Field and Tunelling Centres (CRC, Boca Raton, 1999).

[14] J. Kitagawa, N. Takeda, and M. Ishikawa, Phys. Rev. B 53, 5101 (1996).

[15] R. Caciuffo, J. Paixão, C. Detlefs, M. Longfield, P. Santini, N. Bernhoeft, J. Rebizant, and G. Lander, J. Phys.: Condens. Matter 15, S2287 (2003).

[16] O. Suzuki, H. S. Suzuki, H. Kitazawa, G. Kido, T. Ueno, T. Yamaguchi, Y. Nemoto, and T. Goto, J. Phys. Soc. Jpn. 75, 013704 (2005).

[17] Y. Kuramoto, H. Kusunose, and A. Kiss, J. Phys. Soc. Jpn. 78, 072001 (2009).

[18] S. Lee, A. Paramekanti, and Y. B. Kim, Phys. Rev. B 91, 041104(R) (2015).

[19] A. Sakai and S. Nakatsuji, J. Phys. Soc. Jpn. 80, 063701 (2011).

[20] M. Koseki, Y. Nakanishi, K. Deto, G. Koseki, R. Kashiwazaki, F. Shichinomiya, M. Nakamura, M. Yoshizawa, A. Sakai, and S. Nakatsuji, J. Phys. Soc. Jpn. 80, SA049 (2011).

[21] A. Sakai and S. Nakatsuji, J. Phys.: Conf. Ser. 391, 012058 (2012).

[22] T. J. Sato, S. Ibuka, Y. Nambu, T. Yamazaki, T. Hong, A. Sakai, and S. Nakatsuji, Phys. Rev. B 86, 184419 (2012).

[23] T. Onimaru and H. Kusunose, J. Phys. Soc. Jpn. 85, 082002 (2016).

[24] T. Onimaru, K. T. Matsumoto, Y. F. Inoue, K. Umeo, T. Sakakibara, Y. Karaki, M. Kubota, and T. Takabatake, Phys. Rev. Lett. 106, 177001 (2011).
[25] Y. Shimura, Y. Ohta, T. Sakakibara, A. Sakai, and S. Nakatsuji, J. Phys. Soc. Jpn. 82, 043705 (2013).

[26] T. Onimaru, N. Nagasawa, K. T. Matsumoto, K. Wakiya, K. Umeo, S. Kittaka, T. Sakakibara, Y. Matsushita, and T. Takabatake, Phys. Rev. B 86, 184426 (2012).

[27] T. Onimaru, K. T. Matsumoto, Y. F. Inoue, K. Umeo, Y. Saiga, Y. Matsushita, R. Tamura, K. Nishimoto, I. Ishii, T. Suzuki et al., J. Phys. Soc. Jpn. 79, 033704 (2010).

[28] A. Sakai, K. Kuga, and S. Nakatsuji, J. Phys. Soc. Jpn. 81, 083702 (2012).

[29] K. Matsubayashi, T. Tanaka, A. Sakai, S. Nakatsuji, Y. Kubo, and Y. Uwatoko, Phys. Rev. Lett. 109, 187004 (2012).

[30] K. Matsubayashi, T. Tanaka, J. Suzuki, A. Sakai, S. Nakatsuji, K. Kitagawa, Y. Kubo, and Y. Uwatoko, Proceedings of the International Conference on Strongly Correlated Electron Systems (SCES2013) [JPS Conf. Proc. 3, 011077 (2014)].

[31] M. Tsujimoto, Y. Matsumoto, T. Tomita, A. Sakai, and S. Nakatsuji, Phys. Rev. Lett. 113, 267001 (2014).

[32] K. Iwasa, K. T. Matsumoto, T. Onimaru, T. Takabatake, J.-M. Mignot, and A. Gukasov, Phys. Rev. B 95, 155106 (2017).

[33] T. Taniguchi, M. Yoshida, H. Takeda, M. Takigawa, M. Tsujimoto, A. Sakai, Y. Matsumoto, and S. Nakatsuji, J. Phys. Soc. Jpn. 85, 113703 (2016).

[34] H. Kusunose and T. Onimaru, J. Phys.: Conf. Ser. 592, 012099 (2015).

[35] T. Onimaru, K. Izawa, K. T. Matsumoto, T. Yoshida, Y. Machida, T. Ikeura, K. Wakiya, K. Umeo, S. Kittaka, K. Araki, T. Sakakibara, and T. Takabatake, Phys. Rev. B 94, 075134 (2016).

[36] A. Wörl, T. Onimaru, Y. Tokiwa, Y. Yamane, K. T. Matsumoto, T. Takabatake, and P. Gegenwart, Phys. Rev. B 99, 081117(R) (2019).

[37] O. I. Motrunich, Phys. Rev. B 72, 045105 (2005).

[38] O. I. Motrunich and M. P. A. Fisher, Phys. Rev. B 75, 235116 (2007).

[39] D. N. Sheng, O. I. Motrunich, S. Trebst, E. Gull, and M. P. A. Fisher, Phys. Rev. B 78, 054520 (2008).

[40] D. N. Sheng, O. I. Motrunich, and M. P. A. Fisher, Phys. Rev. B 79, 205112 (2009).

[41] T. Grover, N. Trivedi, T. Senthil, and P. A. Lee, Phys. Rev. B 81, 245121 (2010).

[42] L. de'Medici, A. Georges, and S. Biermann, Phys. Rev. B 72, 205124 (2005). 
[43] L. de'Medici, S. R. Hassan, M. Capone, and X. Dai, Phys. Rev. Lett. 102, 126401 (2009).

[44] F. Freyer, J. Attig, S. B. Lee, A. Paramekanti, S. Trebst, and Y. B. Kim, Phys. Rev. B 97, 115111 (2018).

[45] S. B. Lee, S. Trebst, Y. B. Kim, and A. Paramekanti, Phys. Rev. B 98, 134447 (2018).

[46] A. S. Patri, A. Sakai, S. Lee, A. Paramekanti, S. Nakatsuji, and Y. B. Kim, Nat. Commun. 10, 4092 (2019).

[47] A. S. Patri, I. Khait, and Y. B. Kim, Phys. Rev. Research 2, 013257 (2020).

[48] E. Mendive-Tapia and J. B. Staunton, Phys. Rev. Lett. 118, 197202 (2017).

[49] Y. Akagi, M. Udagawa, and Y. Motome, Phys. Rev. Lett. 108, 096401 (2012).

[50] R. Shiina, H. Shiba, and P. Thalmeier, J. Phys. Soc. Jpn. 66, 1741 (1997).
[51] T. Taniguchi, K. Hattori, M. Yoshida, H. Takeda, S. Nakamura, T. Sakakibara, M. Tsujimoto, A. Sakai, Y. Matsumoto, S. Nakatsuji et al., J. Phys. Soc. Jpn. 88, 084707 (2019).

[52] S. Kittaka, T. Taniguchi, K. Hattori, S. Nakamura, T. Sakakibara, M. Takigawa, M. Tsujimoto, A. Sakai, Y. Matsumoto, and S. Nakatsuji, J. Phys. Soc. Jpn. 89, 043701 (2020).

[53] K. Hattori and H. Tsunetsugu, J. Phys. Soc. Jpn. 83, 034709 (2014).

[54] K. Hattori and H. Tsunetsugu, J. Phys. Soc. Jpn. 85, 094001 (2016).

[55] E. W. Carlson, K. A. Dahmen, E. Fradkin, and S. A. Kivelson, Phys. Rev. Lett. 96, 097003 (2006).

[56] D. S. Caplan, V. Orlyanchik, M. B. Weissman, D. J. Van Harlingen, E. H. Fradkin, M. J. Hinton, and T. R. Lemberger, Phys. Rev. Lett. 104, 177001 (2010). 